\title{
Matrix product state formulation of the multiconfiguration time-dependent Hartree theory
}

\author{
Yuki Kurashige \\ Department of Chemistry, Graduate School of Science, Kyoto University, \\ Kitashirakawa Oiwake-cho, Sakyo-ku Kyoto, 606-8502, Japan
}

(Dated: November 8, 2018)

\begin{abstract}
A matrix product state formulation of the multiconfiguration time-dependent Hartree (MPS-MCTDH) theory is presented. The Hilbert space that is spanned by the direct products of the phonon degree of freedoms, which is linearly parameterized in the MCTDH ansatz and thus results in an exponential increase of the computational cost, is parametrized by the MPS form. Equations of motion based on the Dirac-Frenkel time-dependent variational principle is derived by using the tangent space projection and the projector-splitting technique for the MPS, which have been recently developed. The mean-field operators, which appear in the equation of motion of the MCTDH single particle functions (SPF), are written in terms of the MPS form and efficiently evaluated by a sweep algorithm that is similar to the DMRG sweep. The efficiency and convergence of the MPS approximation to the MCTDH are demonstrated by quantum dynamics simulations of extended excitonic molecular systems.
\end{abstract}

\section{INTRODUCTION}

Matrix product state (MPS) is one of the most successful tensor-network states (TNS) which encode quantum states in the exponentially growing Hilbert space of strongly correlated systems to a sequential product of tensors and was first introduced by White [1, 2] as an eigensolver named as the density-matrix renormalized group (DMRG) algorithm. Although the DMRG works best for one-dimensional Hamiltonians, it has been successfully extended to many fields of application such as ab initio Hamiltonian of quantum chemistry where all the degree of freedoms (DOFs), i.e. electrons, are coupled complexly through the Coulomb interaction [3, 4]. Extension to time-dependent simulations, i.e. time evolution of the MPS, has been also developed, such as the adaptive time-dependent DMRG ( $t$-DMRG) [5, 6] and the time-evolving block-decimation (TEBD) algorithm [7]. The use of the Suzuki-Trotter decomposition by splitting the summation of terms of interactions in the Hamiltonian makes the methods particularly efficient, but in principle it works well for Hamiltonians consisting of local interactions, typically nearest neighbor interaction models.

Recently, time-evolution of the MPS wavefunction has been formulated within the Dirac-Frenkel time-dependent variational principle (TDVP) 8 -10]. This method, in principle, is not restricted to any particular type of Hamiltonians and reasonably applicable to long-range interactions including two-dimensional systems. Efficiency of the method was, however, deteriorated by numerical instability problems arose from the highly nonlinear parametrization in the wavefunction ansatz. It should be noted that there are other closely-related works, e.g. Ref [11 16]. Very recently, Haegeman and co-workers have developed a novel method that circumvents those problems [17].
The method utilizes Lie-Trotter splitting of the tangent space projectors of different sites canonical form, which realizes an efficient and stable propagation of the MPS wavefunctions. The development of robust time evolution method based on the TDVP should significantly expand the applicability of the MPS to a variety of problems. 18 20]

Time evolution methods based on the TDVP have great affinity for molecular quantum dynamics simulations, in which the interactions in the Hamiltonian are represented in the first quantization form. The basis function expansion of the wavefunctions allows us to efficiently evaluate the Hamiltonian matrix elements by analytical or numerical integrations in the real space. The multiconfiguration time-dependent Hartree (MCTDH) theory 21 23 is the most widely used method in this field. In the method, variational space of a vibrational wavefunction is spanned by direct products of one-particle basis functions, of which the shapes are also regarded as variational parameters and evolved with time. While the use of variable one-particle basis significantly reduces the size of the Hilbert space and makes the method highly efficient, 24, 25] the number of linear parameters of the space still grows exponentially with the number of phonon modes. It is, therefore, natural to introduce TNS ansatzes instead of the linear parametrization, in fact the multi-layer formulation (ML-MCTDH), which corresponds to the hierarchical Tucker tensor decomposition 26] and can avoid the expensive exponential cost, 27, 28] made a great success 27, 29, 30] in many fields of application, 31 34] in particular for encoding a tremendous number of harmonic oscillators describing the bath modes that couple to one of the molecules in molecular aggregates. 35 38] The structure of the ML-MCTDH wavefunction is related to the tree tensor network state, 39] which is a generalization of the MPS. In 
fact, a different form of the equation of motion for the MPS-MCTDH wavefunction ansatz can be derived in the framework of the multi-layer formulation (see Appendix $\mathrm{A}$ and $\mathrm{B})$.

\section{THEORY}

In this work, a MPS formulation of the MCTDH theory (MPS-MCTDH) is presented. It is expected to be applied to extended molecular systems where many phonons are strongly and complexly correlated via the electronic inter-state couplings as is often the case with molecular systems. In the MPS-MCTDH method, the molecular wavefunction is parametrized as

$$
|\Psi\rangle=\sum_{\alpha}|\alpha\rangle\left|\Psi^{\alpha}\right\rangle=\sum_{\alpha}|\alpha\rangle \sum_{J} A_{J}^{\alpha}\left|\Phi_{J}^{\alpha}\right\rangle,
$$

where

$$
\begin{aligned}
A_{J\left(=\left\{j_{1}, \cdots, j_{f}\right\}\right)}^{(\alpha)} & \equiv \sum_{\tau_{1} \cdots \tau_{f-1}} a_{\tau_{1}}^{j_{1}} a_{\tau_{1} \tau_{2}}^{j_{2}} \cdots a_{\tau_{f-1}}^{j_{f}}, \\
\left|\Phi_{J}^{\alpha}\right\rangle & \equiv\left|\varphi_{j_{1}}^{(1)} \varphi_{j_{2}}^{(2)} \cdots \varphi_{j_{f}}^{(f)}\right\rangle
\end{aligned}
$$

$\alpha$ denotes an electronic states in the multiset formalism and thus satisfies $\langle\alpha \mid \beta\rangle=\delta_{\alpha \beta}$ [24], $a_{\tau_{p-1} \tau_{p}}^{j_{p}}$ is a site function of the MPS, and $\varphi_{j_{p}}^{(p)}$ is a site basis states which is expressed as a linear combination of the primitive functions as $\sum_{r} c_{j r} \chi_{r}^{p}\left(Q_{p}\right)$ where $c_{j r}$ are variational parameters as well as the site functions $a_{\tau_{p}-1 \tau_{p}}^{j_{p}}$. In constast to the linear coefficients $A_{J}^{(\alpha)}$, of which the dimension grows rapidly as $O\left(n^{f}\right)$ where $n$ is the number of the site basis per site $j_{p}=1 \cdots n$, the dimension of the MPS site functions $\left\{a_{\tau_{p-1} \tau_{p}}^{j_{p}}\right\}$ grows as only $O\left(n m^{2} f\right)$ where $m$ is the bond dimension of each site functions, $\tau_{p}=1 \cdots m$. Note that the high-dimensional $A_{J}^{(\alpha)}\left(O\left(n^{f}\right)\right)$ are never explicitly constructed in the MPS-MCTDH method. In the current implementation, it is possible to combine several phonon modes into single site in the MPS, similarly to the multimode single-particle function $Q_{p}=\left(q_{i}, q_{j}, \cdots\right)$ of the MCTDH method.

Time-evolution of the variational parameters based on the TDVP is formulated by using the tangent space projector $\hat{\mathcal{P}}_{[\Psi]}$

$$
|\dot{\Psi}\rangle=-i \hat{\mathcal{P}}_{[\Psi]} \hat{H}|\Psi\rangle
$$

The tangent space projector acts as orthogonal projection for an arbitrary vector onto the tangent plane, i.e. within the variational space, at the current point $\Psi(t)$; thus, the time-dependent Schrödinger equation is satisfied at the first-order with respect to all the variational parameters. The tangent space projector is expressed as a summation of subspace projectors that must be orthogonal to each other. As the subspace projectors for the MPS site coefficients, the author adopted the projector that imposes the left-gauge fixing condition developed in Ref 17 ]

$$
\begin{aligned}
\hat{\mathcal{P}}_{\mathrm{MPS}} & =\sum_{p=1}^{f} \hat{\mathcal{P}}_{p}^{+}-\sum_{p=1}^{f-1} \hat{\mathcal{P}}_{p}^{-}, \\
\hat{\mathcal{P}}_{p}^{+} & \equiv \sum_{\lambda_{p-1} j_{p} \lambda_{p}}\left|\Psi_{\lambda_{p-1}}^{\mathrm{L}} \varphi_{j_{p}}^{(p)} \Psi_{\lambda_{p}}^{\mathrm{R}}\right\rangle\left\langle\Psi_{\lambda_{p}}^{\mathrm{R}} \varphi_{j_{p}}^{(p)} \Psi_{\lambda_{p-1}}^{\mathrm{L}}\right|, \\
\hat{\mathcal{P}}_{p}^{-} & \equiv \sum_{\gamma_{p} \lambda_{p}}\left|\Psi_{\gamma_{p}}^{\mathrm{L}} \Psi_{\lambda_{p}}^{\mathrm{R}}\right\rangle\left\langle\Psi_{\lambda_{p}}^{\mathrm{R}} \Psi_{\gamma_{p}}^{\mathrm{L}}\right|,
\end{aligned}
$$

and

$$
\begin{aligned}
\left|\Psi_{\tau_{p}}^{\mathrm{L}}\right\rangle & \equiv \sum_{\tau_{1} \cdots \tau_{p-1}} L_{\tau_{1}}^{j_{1}} \cdots L_{\tau_{p-1} \tau_{p}}^{j_{p}}\left|\varphi_{j_{1}}^{(1)} \cdots \varphi_{j_{p}}^{(p)}\right\rangle \\
\left|\Psi_{\tau_{p}^{\prime}}^{\mathrm{R}}\right\rangle & \equiv \sum_{\tau_{p+1} \cdots \tau_{f-1}} R_{\tau_{p}^{\prime} \tau_{p+1}}^{j_{p+1}} \cdots R_{\tau_{f-1}}^{j_{f}}\left|\varphi_{j_{p+1}}^{(p+1)} \cdots \varphi_{j_{f}}^{(f)}\right\rangle
\end{aligned}
$$

where $L_{\tau_{p-2} \tau_{p-1}}^{j_{p-1}}$ and $R_{\tau_{p} \tau_{p+1}}^{j_{p+1}}$ denote the left-orthonormal and right-orthonormal site functions, respectively, appearing in the $p$-canonical form of MPS wavefunctions

$$
A_{J}^{(\alpha)}=\sum_{\tau_{1} \cdots \tau_{f-1}} L_{\tau_{1}}^{j_{1}} \cdots L_{\tau_{p-2} \tau_{p-1}}^{j_{p-1}} C_{\tau_{p-1} \tau_{p}}^{j_{p}} R_{\tau_{p} \tau_{p+1}}^{j_{p+1}} \cdots R_{\tau_{f-1}}^{j_{f}},
$$

which can be transformed to the next site $(p+1)$-canonical form by using the relation

$$
\begin{aligned}
\sum_{\tau_{p}^{\prime}} C_{\tau_{p-1} \tau_{p}^{\prime}}^{j_{p}} R_{\tau_{p}^{\prime} \tau_{p+1}}^{j_{p+1}} & =\sum_{\tau_{p}^{\prime} \tau_{p}} L_{\tau_{p-1} \tau_{p}}^{j_{p}} \sigma_{\tau_{p} \tau_{p}^{\prime}} R_{\tau_{p}^{\prime} \tau_{p+1}}^{j_{p+1}} \\
& =\sum_{\tau_{p}} L_{\tau_{p-1} \tau_{p}}^{j_{p}} C_{\tau_{p} \tau_{p+1}}^{j_{p+1}}
\end{aligned}
$$

where

$$
\sigma_{\tau_{p} \tau_{p}^{\prime}} \equiv \sum_{j_{p}^{\prime} \tau_{p-1}^{\prime}} L_{\tau_{p-1}^{\prime} \tau_{p}}^{j_{p}^{\prime}} C_{\tau_{p-1}^{\prime} \tau_{p}^{\prime}}^{j_{p}^{\prime}},
$$

because $L_{\tau_{p-1} \tau_{p}}^{j_{p}}$ is obtained by the diagonalization of

$$
\sum_{\tau_{p}} C_{\tau_{p-1}^{\prime} \tau_{p}}^{j_{p}^{\prime}} \bar{C}_{\tau_{p-1} \tau_{p}}^{j_{p}}=\sum_{\tau_{p}} L_{\tau_{p-1}^{\prime} \tau_{p}}^{j_{p}^{\prime}} w_{\tau_{p}}^{(\mathrm{L})} \bar{L}_{\tau_{p-1} \tau_{p}}^{j_{p}}
$$

for details refer to literature, e.g. Ref[40]. In the case of the MPS-MCTDH ansatz, this projector generates not only the variation of site functions of the MPS, but also the variation of site basis states, themselves. It should bring a complication for its formulation, thus a certain MCTDH gauge, $\left\langle\varphi_{j}^{(p)} \mid \dot{\varphi}_{l}^{(p)}\right\rangle=0$, is adopted, by which the variation of the site basis states generated by $\hat{\mathcal{P}}_{\mathrm{MPS}}$ are vanished. The projector for the complementary space 
$\hat{\mathcal{P}}^{\prime} \equiv \hat{\mathcal{P}}-\hat{\mathcal{P}}_{\mathrm{MPS}}$, i.e. the site basis functions space, can be derived in the same way as Ref 41 43. except that the MCTDH coefficient is replaced by the MPS, and the Lie-Trotter splitting of the tangent space projectors should be done naturally for all the projector

$$
e^{-i \hat{\mathcal{P}} \hat{H} 2 \delta} \approx e^{-i \hat{\mathcal{P}}^{\prime} \hat{H} \delta} e^{-i \hat{\mathcal{P}}_{\mathrm{MPS}} \hat{H} \delta} e^{-i \hat{\mathcal{P}}^{\prime} \hat{H} \delta} .
$$

Another way is to assume the constant mean-field (CMF) approximation, 44 in which the mean-field operators and the integrals written in the site basis functions are frozen during each step of the propagation, and thus the time-evolution of the MPS site functions and the site basis functions are decoupled during the step intervals. The CMF integration method, which was adopted in this work, becomes advantageous for extended systems where the computation of the mead-field operators and related operations are the most time-consuming steps.

Another issue of discussion for using the MPS form in the MCTDH theory is the construction of mean-field operators for single-particle functions $\left\{\varphi_{j_{p}}^{(p)}\right\}$ expressed as

$$
\langle\hat{O}\rangle_{j k}^{\alpha \beta(p)}=\left\langle\Psi_{j}^{\alpha(p)}|\hat{O}| \Psi_{k}^{\beta(p)}\right\rangle
$$

where

$$
\begin{aligned}
\left|\Psi_{k}^{\beta(p)}\right\rangle \equiv \sum_{k_{1}} & \cdots \sum_{k_{p-1}} \sum_{k_{p+1}} \cdots \sum_{k_{f}} A_{k_{1} \cdots k_{p-1} k k_{p+1}}^{(\beta)} \\
& \times\left|\varphi_{k_{1}}^{(1)} \cdots \varphi_{k_{p-1}}^{(p-1)} \varphi_{k_{p+1}}^{(p+1)} \cdots \varphi_{k_{f}}^{(f)}\right\rangle .
\end{aligned}
$$

In general, phonon modes of molecular systems are complexly coupled through the potential energy surface created by the electronic-state; thus, there can be a $f$-body interaction $\hat{V}\left(Q_{1}, \cdots, Q_{f}\right)$ term in the Hamiltonian, but in many cases the $f$-body interaction is efficiently expanded by the $n$-mode coupling representation [45] and usually it is sufficient to truncate up to the fourth order expansion [46]. An efficient evaluation of three and four-body operators for MPS wavefunction by the DMRG sweep algorithm is presented in our previous work [47]. Alternatively, the $f$-body Hamiltonian are reduced to products of one-body operators of the single site basis $\varphi^{(p)}\left(Q_{p}\right)$ in the MCTDH method [23] as

$$
\begin{aligned}
& \left\langle\varphi_{j_{1}}^{(1)} \cdots \varphi_{j_{f}}^{(f)}\left|\hat{O}^{(1, . ., f)}\right| \varphi_{k_{1}}^{(1)} \cdots \varphi_{k_{f}}^{(f)}\right\rangle \\
& =\sum_{a} c_{a}\left\langle\varphi_{j_{1}}^{(1)}\left|\hat{o}_{a}^{(1)}\right| \varphi_{k_{1}}^{(1)}\right\rangle \cdots\left\langle\varphi_{j_{f}}^{(f)}\left|\hat{o}_{a}^{(f)}\right| \varphi_{k_{f}}^{(f)}\right\rangle
\end{aligned}
$$

This product form is also very suitable for the MPS wavefunction. For example, a mean-field operator in the product form is decomposed as

$$
\begin{aligned}
\left\langle\hat{O}_{a}\right\rangle_{j k}^{(p)} & =\bar{C}_{\lambda_{p-1} \lambda_{p}}^{j}\left\langle\Psi_{\lambda_{p-1}}^{\mathrm{L}}\left|\hat{O}_{a}^{(1, . ., p-1)}\right| \Psi_{\tau_{p-1}}^{\mathrm{L}}\right\rangle \\
& \times\left\langle\Psi_{\lambda_{p}}^{\mathrm{R}}\left|\hat{O}_{a}^{(p+1, \ldots, f)}\right| \Psi_{\tau_{p}}^{\mathrm{R}}\right\rangle C_{\tau_{p-1} \tau_{p}}^{k} \hat{o}_{a}^{(p)} .
\end{aligned}
$$

The operators in the left and right blocks can be prepared easily in the same way as the DMRG algorithm, and the mean-field operators for the different sites $p$ are constructed by the $p$-canonical form transformation along the MPS lattice.

\section{RESULTS AND DISCUSSION}

To demonstrate the efficiency of the MPS-MCTDH method, exciton-phonon dynamics in the molecular aggregates were performed. The applications to the exciton-phonon dynamics are important for computing the quantum efficiency of energy conversion and transfer in molecular aggregates, [48 51] e.g. photovoltaic and photosynthetic systems, but application to the extended systems were hampered by the exponential increase of the computational cost with the number of vibrational degree of freedoms. Most recently, Ren and co-worker successfully computed the absorption and fluorescence spectra of molecular aggregates with a time-dependent DMRG algorithm at both zero and finite temperature. 52 The Hamiltonian adopted for the benchmark simulation is

$$
\begin{aligned}
\hat{H} & =\sum_{\alpha}|\alpha\rangle\langle\alpha| \sum_{v} \frac{\omega_{v}^{\alpha}}{2}\left(-\frac{\partial^{2}}{\partial Q_{v}^{2}}+\hat{Q}_{v}^{2}\right) \\
& +\sum_{\alpha}|\alpha\rangle\left\langle\alpha\left|\sum_{v} \hat{Q}_{v} \kappa_{v}^{\alpha}+\sum_{\alpha \beta}\right| \alpha\right\rangle\langle\beta| J_{\alpha \beta},
\end{aligned}
$$

where $\omega_{v}^{\alpha}$ is the harmonic frequency of the phonon mode $v$ on the diabatic electronic-state $\alpha, \kappa_{v}^{\alpha}$ is the first-order coupling between the diabatic electronic-state $\alpha$ and the phonon mode $v$, and $J_{\alpha \beta}$ for $\alpha \neq \beta$ is the diabatic coupling between $\alpha$ and $\beta$ states and that for $\alpha=\beta$ is the energy gaps between the states at the origin, $Q_{v}=0$ for all $v$. The diabatic electronic-state of the molecular aggregate, $|\alpha\rangle$, is characterized by the electronic state of each molecules; if the $i$-th molecule is in its excited-state, e.g. $S_{1}$ state, while all the other molecules are in the ground state, the state is denoted by $|i\rangle$. The parameters in the Hamiltonian, $\omega_{v}^{\alpha}, \kappa_{v}^{\alpha}$, and $J_{\alpha \beta}$, can be quantitatively determined by ab initio quantum chemical calculations to simulate real molecular systems. In the following, a single local vibrational mode per monomer is considered; thus the number of phonon DOFs is equal to the number of molecules and to the number of electronic states. The dimension for the site basis functions is set to four for all the phonon DOFs, and each site basis function is expressed 
by a linear combination of eight eigenfunctions of the harmonic oscillator from the lowest quantum number. The MCTDH and MPS-MCTDH methods described in this work were implemented in Python3.

Figure 1 shows the population dynamics of an exciton in a molecular aggregate consisting of eight molecules 1-D aligned and sixteen molecules 2-D aligned. The population of the electronic-state $|i\rangle$ in which the exciton is localized on the $i$-th molecule from the end is calculated as $\rho_{i}(t)=\langle\Psi \mid i\rangle\langle i \mid \Psi\rangle=\left\langle\Psi^{i} \mid \Psi^{i}\right\rangle$. At time $t=0$, only the monomer at one end of the 1-D aggregate is electronically excited, and time evolution of the population of the exciton on the opposite end monomer are shown. The values for the parameters are $\omega_{v}^{\alpha}=1255 \mathrm{~cm}^{-1}$ and $\kappa_{v}^{\alpha}=1.072 \times \omega_{v}^{\alpha}$, typical values for intramolecular mode of organic semiconductors. The electronic states in which the excitons are located at the nearest neighbor to each other interact with each other by the diabatic coupling $J$ in 1-D and 2-D systems.

A multiset time-dependent Hartree (TDH) method, in which each vibrational wavefunction $\Psi^{\alpha}$ associated with an electronic-state is expressed by a Hartree product, hence the vibrational DOFs are non-entangled, are also
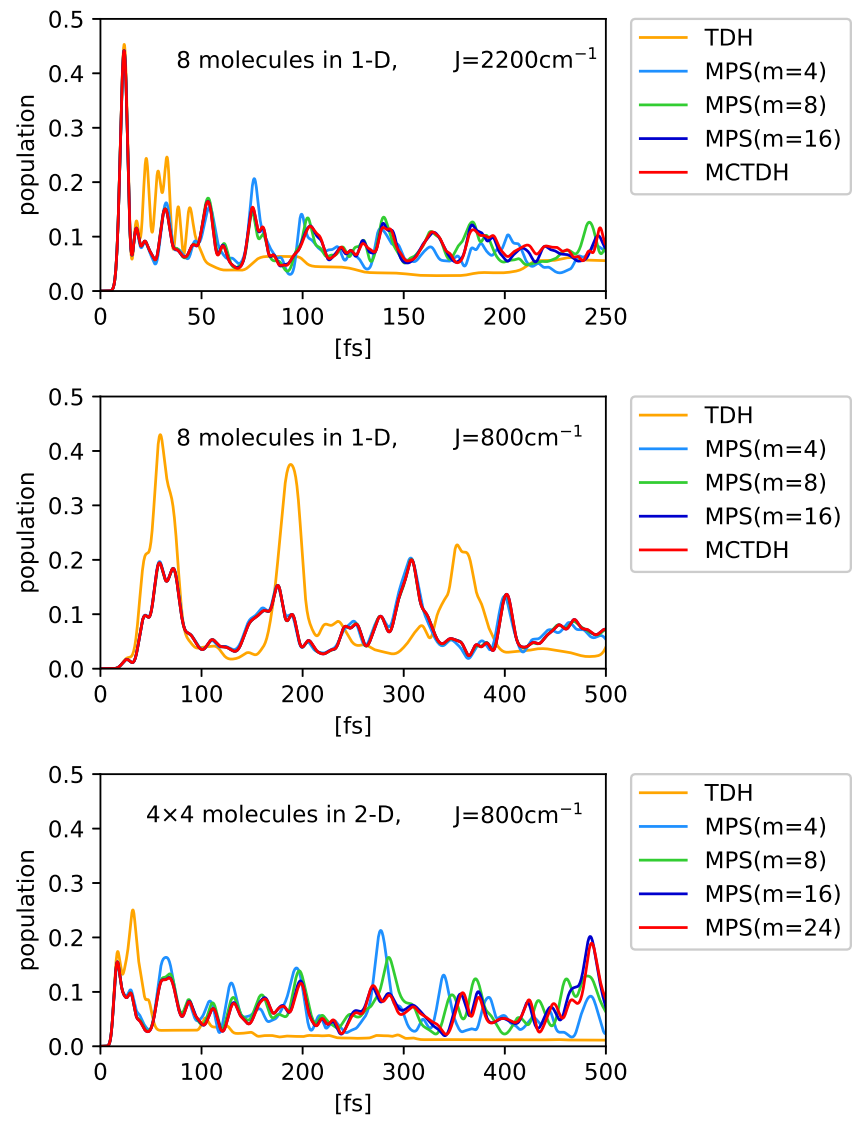

FIG. 1. Time evolution of the exciton population with various methods for the 1-D and 2-D systems. The populations at the opposite end of the initially populated site are shown.
TABLE I. Elapsed CPU time (sec) for computing the MPS related operations, i.e. computing the mean-field operators and propagation of the MPS site functions, in a step of the CMF integration algorithm. The timings for the corresponding operations in the MCTDH method are also shown.

\begin{tabular}{cccccc}
\hline \hline \multirow{2}{*}{$\begin{array}{c}\text { \# of } \\
\text { molecules }\end{array}$} & $\begin{array}{c}\text { MCTDH } \\
\text { method }\end{array}$ & \multicolumn{4}{c}{ MPS-MCTDH method } \\
\cline { 3 - 6 } & $m=4$ & $m=8$ & $m=16$ & $m=32$ \\
\hline 8 & 5.9 & 1.1 & 1.4 & 2.4 & 5.9 \\
10 & 224 & 1.8 & 2.2 & 4.2 & 12 \\
12 & 16907 & 2.5 & 3.3 & 6.8 & 22 \\
16 & - & 4.8 & 6.7 & 14 & 53 \\
24 & - & 13 & 19 & 45 & 179 \\
32 & - & 27 & 42 & 99 & 421 \\
64 & - & 270 & 415 & 1013 & 4638 \\
96 & - & 859 & 1373 & 3372 & 15509 \\
\hline \hline
\end{tabular}

performed for comparison. In all cases, the deviations between the $\mathrm{TDH}$ and $\mathrm{MCTDH}$ methods are found to be significant, which can be regarded as a measure of the strength of the entanglement between the vibrational DOFs in the system. The earliest maximum of the population in the plots corresponds to the first arrival of the exciton from the initial location, i.e. reflects the mobility of excitons, and the waves should go back and forth causing interference with each other. For the 1-D system with the $J=800 \mathrm{~cm}^{-1}$ (top panel), the MPS well reproduces the results of the MCTDH method, even with the smallest bond dimensions, $m=4$. For the same 1-D system with $J=2200 \mathrm{~cm}^{-1}$ (middle panel), the $\operatorname{MPS}(m=4)$ plots deviates from the MCTDH plots after 70 fs for. The $\operatorname{MPS}(m=8)$ is more robust and can trace the MCTDH plot and slightly deviate after 200 fs. The MPS with the largest bond dimensions $m=16$ reproduces the $\mathrm{MCTDH}$ result. It suggests that the required size of the bond dimension $m$ to maintain the same accuracy depends on the strength of the coupling. For the 2-D system with $J=800 \mathrm{~cm}^{-1}$ (bottom panel), the convergence of population dynamics to the result with large $m$ is slower than that for the 1-D system with the same coupling strength. Due to the nature of the MPS, which is a sequential product of tensors, the required size of $m$ is increased for 2-D and higher dimensional systems.

Table [ shows the elapsed CPU time for computing the mean-field operators and propagation of the MCTDH coefficients $A_{J}$ or the MPS site functions $a_{\tau_{1}}^{j_{1}}, a_{\tau_{1} \tau_{2}}^{j_{2}}, \cdots, a_{\tau_{f-1}}^{j_{f}}$, by the short-time Lanczos algorithm, per one step of the CMF integration algorithm, in which the mean-filed operators are evaluated two times and the coefficient are propagated three times including the backward propagation for the error estimation 44]. Due to the linear parametrization of the Hilbert space, the elapsed time for the MCTDH 
grows exponentially with the number of the molecules. The formal computational scaling of the MPS is $O\left(n m^{3} k^{2} f\right)$, where $k$ is the number of electronic states, for the evaluation of the mean-field operators and the time propagation of the site functions. In this excitonic systems, the number of vibrational DOFs $f$ and electronic states $k$ grows linearly with the number of molecules $N$, thus the formal computational scaling with respect to $N$ is cubic when $n$ and $m$ are constant, and that with $m$ is also cubic. The actual scaling with $m$ between $m=8$ and $m=16$ is linear, and that between $m=16$ and $m=32$ is quadratic, i.e. smaller than the formal scaling. This is because the most time consuming steps are relatively small matrix-matrix multiplication; typically the tensor contraction in Eq.15), in which the matrix size for the matrix-matrix multiplication is $m \times m$, and high throughput cannot be achieved by the current implementation for small $m$.

To summarize, A matrix product state formulation of the multiconfiguration time-dependent Hartree is presented. The MPS can efficiently encode the Hilbert space of the phonon DOFs, which grows exponentially with the number of modes. Due to the nature of the MPS form, it is particularly effective for extended systems and modes that are strongly correlated as is often the case with molecular systems. The efficiency of the method was demonstrated on the quantum dynamics of the extended excitonic systems.

\section{ACKNOWLDGEMENTS}

The author thanks Yoshitaka Tanimura, Tatsushi Ikeda, and Takeshi Sato for many valuable discussions.

\section{APPENDIX A: ALGORITHM FOR PROPAGATING THE MPS SITE COEFFICIENTS}

The MPS site coefficients are propagated sequentially from the leftmost site $p=1$ to the rightmost site $p=f$, in the former half, and then proceeds in the opposite direction, i.e. from the site $p=f$ to the site $p=1$ in the latter half of the algorithm, to accomplish the time propagation written by

$$
\begin{aligned}
& |\Psi(\mathrm{t}+2 \delta)\rangle=e^{-i \hat{\mathcal{P}}_{\mathrm{MPS}} \hat{H} 2 \delta}|\Psi(\mathrm{t})\rangle \\
& =e^{-i\left(\hat{\mathcal{P}}_{1}^{+}-\hat{\mathcal{P}}_{1}^{-}+\cdots-\hat{\mathcal{P}}_{f-1}^{-}+2 \hat{\mathcal{P}}_{f}^{+}-\hat{\mathcal{P}}_{f-1}^{-}+\cdots-\hat{\mathcal{P}}_{1}^{-}+\hat{\mathcal{P}}_{1}^{+}\right) \hat{H} \delta}|\Psi(\mathrm{t})\rangle \\
& \approx e^{-i \hat{\mathcal{P}}_{1}^{+} \hat{H} \delta} e^{+i \hat{\mathcal{P}}_{1}^{-} \hat{H} \delta} \cdots e^{+i \hat{\mathcal{P}}_{f-1}^{-} \hat{H} \delta} e^{-i \hat{\mathcal{P}}_{f}^{+} \hat{H} \delta} \\
& \times e^{-i \hat{\mathcal{P}}_{f}^{+} \hat{H} \delta} e^{+i \hat{\mathcal{P}}_{f-1}^{-} \hat{H} \delta} \cdots e^{+i \hat{\mathcal{P}}_{1}^{-} \hat{H} \delta} e^{-i \hat{\mathcal{P}}_{1}^{+} \hat{H} \delta}|\Psi(\mathrm{t})\rangle \cdot
\end{aligned}
$$

The time integrations based on the symmetric expansion are expected to have a favorable error of order $\mathcal{O}\left(\delta^{3}\right)$. In the following, the index $\alpha$ that denotes electronic states is omitted for clarity.
0 ) At the beginning, the MPS wavefunction is prepared in the $[p=1]$-canonical form, i.e. the coefficients except for the site $p=1$ are right-orthonormal,

$$
\sum_{\tau, J} C_{\tau_{1}}^{j_{1}} R_{\tau_{1} \tau_{2}}^{j_{2}} \cdots R_{\tau_{f-2} \tau_{f-1}}^{j_{f-1}} R_{\tau_{f-1}}^{j_{f}}\left|\Phi_{J}\right\rangle,
$$

which is always available through the gauge transformation by Eq. (8) if necessary.

1) In this step, the coefficients for the current site (starts with $p=1), C_{\tau_{p-1} \tau_{p}}^{j_{p}}$, are propagated by applying the operator exponential $e^{-i \hat{\mathcal{P}}_{p}^{+} \hat{H} \delta}$ to the wavefunction as

$$
\begin{aligned}
C_{\tau_{p-1} \tau_{p}}^{\prime j_{p}}= & \left\langle\Psi_{\tau_{p}}^{\mathrm{R}} \varphi_{j_{p}}^{(p)} \Psi_{\tau_{p-1}}^{\mathrm{L}}\left|e^{-i \hat{\mathcal{P}}_{p}^{+} \hat{H} \delta}\right| \Psi\right\rangle \\
= & \left\langle\Psi_{\tau_{p}}^{\mathrm{R}} \varphi_{j_{p}}^{(p)} \Psi_{\tau_{p-1}}^{\mathrm{L}}\right| e^{-i \hat{\mathcal{P}}_{p}^{+} \hat{H} \delta} \\
& \times \sum_{\tau_{p-1}^{\prime} j_{p}^{\prime} \tau_{p}^{\prime}} C_{\tau_{p-1}^{\prime} \tau_{p}^{\prime}}^{j_{p}^{\prime}}\left|\Psi_{\tau_{p-1}^{\prime}}^{\mathrm{L}} \varphi_{j_{p}^{\prime}}^{(p)} \Psi_{\tau_{p}^{\prime}}^{\mathrm{R}}\right\rangle .
\end{aligned}
$$

It is expressed as

$$
\boldsymbol{c}^{\prime}=e^{-i \boldsymbol{H} \delta} \boldsymbol{c}
$$

where the indices $\left(\tau_{p-1}, j_{p}, \tau_{p}\right)$ of $C_{\tau_{p-1} \tau_{p}}^{j_{p}}$ are flattened to one dimension in the vector $\boldsymbol{c}$ and $\boldsymbol{H}$ is a matrix defined as

$H_{\left(\tau_{p-1}^{\prime} j_{p}^{\prime}, \tau_{p}^{\prime}\right)}^{\left(\tau_{p-1}, j_{p}, \tau_{p}\right)} \equiv\left\langle\Psi_{\tau_{p}}^{\mathrm{R}} \varphi_{j_{p}}^{(p)} \Psi_{\tau_{p-1}}^{\mathrm{L}}|\hat{H}| \Psi_{\tau_{p-1}^{\prime}}^{\mathrm{L}} \varphi_{j_{p}^{\prime}}^{(p)} \Psi_{\tau_{p}^{\prime}}^{\mathrm{R}}\right\rangle$.

The multiplication of the matrix exponential in Eq.(19) is evaluated by an efficient short iterative Lanczos (SIL) integrator, which is one of the Krylov subspace methods and requires only the matrix-vector multiplications $\boldsymbol{v}=\boldsymbol{H} \boldsymbol{c}$. Analogous to the quantum chemical DMRG, of which the Hamiltonian contains four-site operators, 53] the Hamiltonian is decomposed as

$$
\hat{H}=\sum_{i} \hat{\mathcal{O}}_{\mathrm{L}}^{i} \hat{\mathcal{O}}_{\mathrm{C}}^{i} \hat{\mathcal{O}}_{\mathrm{R}}^{i}
$$

where the operator $\hat{\mathcal{O}}_{\mathrm{L}}^{i}, \hat{\mathcal{O}}_{\mathrm{C}}^{i}$, and $\hat{\mathcal{O}}_{\mathrm{R}}^{i}$ act on the phonon modes $Q_{1} \cdots Q_{p-1}, Q_{p}$, and $Q_{p+1} \cdots Q_{f}$, respectively. Now the Hamiltonian multiplication is reduced to

$$
\begin{aligned}
v_{\tau_{p-1} \tau_{p}}^{j_{p}} & =\sum_{\tau_{p-1}^{\prime}, j_{p}^{\prime}, \tau_{p}^{\prime}} H_{\tau_{p-1}^{\prime}, j_{p}^{\prime}, \tau_{p}^{\prime}}^{\tau_{p-1}, j_{p}, \tau_{p}} C_{\tau_{p-1}^{\prime}, \tau_{p}^{\prime}}^{j_{p}^{\prime}} \\
& =\sum_{i} \sum_{\tau_{p-1}^{\prime}}\left\langle\mathcal{O}_{\mathrm{L}}^{i}\right\rangle_{\tau_{p-1}^{\prime}}^{\tau_{p-1}} \sum_{j_{p}^{\prime}}\left\langle\mathcal{O}_{\mathrm{C}}^{i}\right\rangle_{j_{p}^{\prime}}^{j_{p}} \sum_{\tau_{p}^{\prime}}\left\langle\mathcal{O}_{\mathrm{R}}^{i}\right\rangle_{\tau_{p}^{\prime}}^{\tau_{p}} C_{\tau_{p-1}^{\prime}, \tau_{p}^{\prime}}^{j_{p}^{\prime}},
\end{aligned}
$$


where

$$
\begin{aligned}
\left\langle\mathcal{O}_{\mathrm{L}}^{i}\right\rangle_{\tau_{p-1}^{\prime}}^{\tau_{p-1}} & \equiv\left\langle\Psi_{\tau_{p-1}}^{\mathrm{L}}\left|\hat{\mathcal{O}}_{\mathrm{L}}^{i}\right| \Psi_{\tau_{p-1}^{\prime}}^{\mathrm{L}}\right\rangle, \\
\left\langle\mathcal{O}_{\mathrm{C}}^{i}\right\rangle_{j_{p}^{\prime}}^{j_{p}} & \equiv\left\langle\varphi_{j_{p}}^{(p)}\left|\hat{\mathcal{O}}_{\mathrm{C}}^{i}\right| \varphi_{j_{p}^{\prime}}^{(p)}\right\rangle, \\
\left\langle\mathcal{O}_{\mathrm{R}}^{i}\right\rangle_{\tau_{p}^{\prime}}^{\tau_{p}} & \equiv\left\langle\Psi_{\tau_{p}}^{\mathrm{R}}\left|\hat{\mathcal{O}}_{\mathrm{R}}^{i}\right| \Psi_{\tau_{p}^{\prime}}^{\mathrm{R}}\right\rangle .
\end{aligned}
$$

The computational scaling of this step is, therefore, $O\left(n m^{3} \tilde{k}\right)$ where $n, m$, and $\tilde{k}$ are the dimension of $j_{p}$, $\tau_{p(-1)}$, and $i$, respectively. Note that the summation over the phonon modes $v$ in the Hamiltonian [Eq. [16)] is not appeared in the summation over $i$ in Eq.(21) because it has already been taken in the evaluation of $\left\langle\mathcal{O}_{\mathrm{L}(\mathrm{R})}\right\rangle$, e.g.

$$
\begin{aligned}
\sum_{v=1}^{f} g_{v}\left(\hat{Q}_{v}\right) & =\sum_{v=1}^{p-1} g_{v}\left(\hat{Q_{v}}\right) \otimes \mathbb{1}_{\mathrm{C}} \otimes \mathbb{1}_{\mathrm{R}} \\
& +\mathbb{1}_{\mathrm{L}} \otimes g_{p}\left(\hat{Q_{p}}\right) \otimes \mathbb{1}_{\mathrm{R}} \\
& +\mathbb{1}_{\mathrm{L}} \otimes \mathbb{1}_{\mathrm{C}} \otimes \sum_{v=p+1}^{f} g_{v}\left(\hat{Q_{v}}\right)
\end{aligned}
$$

thus $\tilde{k}$ grows only with the number of the electronic states $k$ (c.f. Eq.(16)).

2) The wavefunction after the propagation $C_{\tau_{p-1} \tau_{p}}^{j_{p}} \rightarrow$ $C_{\tau_{p-1} \tau_{p}}^{\prime j_{p}}$ in the previous step is expressed as

$$
\sum_{\tau, J} L_{\tau_{1}}^{\prime j_{1}} \cdots L_{\tau_{p-2} \tau_{p-1}}^{\prime j_{p-1}}\left(C_{\tau_{p-1} \tau_{p}}^{\prime j_{p}}\right) R_{\tau_{p} \tau_{p+1}}^{j_{p+1}} \cdots R_{\tau_{f-1}}^{j_{f}}\left|\Phi_{J}^{\alpha}\right\rangle .
$$

It is transformed to

$$
\sum_{\tau, \gamma_{p}, J} L_{\tau_{1}}^{\prime j_{1}} \cdots L_{\tau_{p-2} \tau_{p-1}}^{\prime j_{p-1}}\left(L_{\tau_{p-1} \gamma_{p}}^{\prime j_{p}} \sigma_{\tau_{p}}^{\prime \gamma_{p}}\right) R_{\tau_{p} \tau_{p+1}}^{j_{p+1}} \cdots R_{\tau_{f-1}}^{j_{f}}\left|\Phi_{J}^{\alpha}\right\rangle .
$$

by using the orthogonal decomposition in Eq. (8)

3) This step is similar to Step 10 and the matrix $\sigma_{\gamma_{p} \tau_{p}}^{\prime}$ is propagated (but backward in time) by applying the operator exponential $e^{+i \hat{\mathcal{P}}_{p}^{-} \hat{H} \delta}$ to the wavefunction as

$$
\begin{aligned}
\sigma_{\tau_{p}}^{\gamma_{p}}= & \left\langle\Psi_{\tau_{p}}^{\mathrm{R}} \Psi_{\gamma_{p}}^{\mathrm{L}}\left|e^{+i \hat{\mathcal{P}}_{p}^{-} \hat{H} \delta}\right| \Psi\right\rangle \\
= & \left\langle\Psi_{\tau_{p}}^{\mathrm{R}} \Psi_{\gamma_{p}}^{\mathrm{L}}\right| e^{+i \hat{\mathcal{P}}_{p}^{-} \hat{H} \delta} \\
& \times \sum_{\gamma_{p}^{\prime} \tau_{p}^{\prime}} \sigma_{\tau_{p}^{\prime}}^{\prime \gamma_{p}^{\prime}}\left|\Psi_{\gamma_{p}^{\prime}}^{\mathrm{L}} \Psi_{\tau_{p}^{\prime}}^{\mathrm{R}}\right\rangle .
\end{aligned}
$$

It can be rewritten as

$$
\boldsymbol{\sigma}=e^{+i \boldsymbol{K} \delta} \boldsymbol{\sigma}^{\prime}
$$

where the indices $\left(\gamma_{p}, \tau_{p}\right)$ of $\sigma_{\tau_{p}}^{\prime \gamma_{p}}$ are flattened to one dimension in the vector $\boldsymbol{\sigma}^{\prime}$ and $\boldsymbol{K}$ is a matrix defined as

$$
K_{\left(\gamma_{p}^{\prime} \tau_{p}^{\prime}\right)}^{\left(\gamma_{p} \tau_{p}\right)} \equiv\left\langle\Psi_{\tau_{p}}^{\mathrm{R}} \Psi_{\gamma_{p}}^{\mathrm{L}}|\hat{H}| \Psi_{\gamma_{p}^{\prime}}^{\mathrm{L}} \Psi_{\tau_{p}^{\prime}}^{\mathrm{R}}\right\rangle .
$$

The multiplication of the matrix exponential in Eq.(26) is evaluated by the SIL, which requires only the matrix-vector multiplication $\boldsymbol{u}=\boldsymbol{K} \boldsymbol{\sigma}$. As done in Step 1 the Hamiltonian is decomposed as

$$
\hat{H}=\sum_{i} \hat{\mathcal{O}}_{\mathrm{L}}^{i} \hat{\mathcal{O}}_{\mathrm{R}}^{i}
$$

where the operator $\hat{\mathcal{O}}_{\mathrm{L}}^{i}$ and $\hat{\mathcal{O}}_{\mathrm{R}}^{i}$ act on the phonon modes $Q_{1} \cdots Q_{p}$ and $Q_{p+1} \cdots Q_{f}$, respectively.

$$
\begin{aligned}
u_{\tau_{p}}^{\gamma_{p}} & =\sum_{\gamma_{p}^{\prime}, \tau_{p}^{\prime}} K_{\gamma_{p}^{\prime}, \tau_{p}^{\prime}}^{\gamma_{p}, \tau_{p}} \sigma_{\tau_{p}^{\prime}}^{\gamma_{p}^{\prime}} \\
& =\sum_{i} \sum_{\gamma_{p}^{\prime}}\left\langle\mathcal{O}_{\mathrm{L}}^{i}\right\rangle_{\gamma_{p}^{\prime}}^{\gamma_{p}} \sum_{\tau_{p}^{\prime}}\left\langle\mathcal{O}_{\mathrm{R}}^{i}\right\rangle_{\tau_{p}^{\prime}}^{\tau_{p}} \sigma_{\tau_{p}^{\prime}}^{\prime \gamma_{p}^{\prime}} .
\end{aligned}
$$

The computational scaling of this step is, therefore, $O\left(m^{3} \tilde{k}\right)$.

4) The wavefunction obtained by the propagation $\sigma_{\tau_{p}}^{\prime \gamma_{p}} \rightarrow$ $\sigma_{\tau_{p}}^{\gamma_{p}}$ in the previous step is expressed as

$\sum_{\tau, \gamma_{p}, J} L_{\tau_{1}}^{\prime j_{1}} \cdots L_{\tau_{p-1} \tau_{p}}^{\prime j_{p}}\left(\sigma_{\gamma_{p}}^{\tau_{p}} R_{\gamma_{p} \tau_{p+1}}^{j_{p+1}}\right) R_{\tau_{p+1} \tau_{p+2}}^{j_{p+2}} \cdots R_{\tau_{f-1}}^{j_{f}}\left|\Phi_{J}^{\alpha}\right\rangle$.

It is transformed to

$$
\sum_{\boldsymbol{\tau}, J} L_{\tau_{1}}^{\prime j_{1}} \cdots L_{\tau_{p-1} \tau_{p}}^{\prime j_{p}}\left(C_{\tau_{p} \tau_{p+1}}^{j_{p+1}}\right) R_{\tau_{p+1} \tau_{p+2}}^{j_{p+2}} \cdots R_{\tau_{f-1}}^{j_{f}}\left|\Phi_{J}^{\alpha}\right\rangle .
$$

5) Steps 14 4are repeated until the current site $p$ reaches the rightmost site $p=f$

$$
\sum_{\tau, J} L_{\tau_{1}}^{\prime j_{1}} L_{\tau_{1} \tau_{2}}^{\prime j_{2}} \cdots L_{\tau_{f-2} \tau_{f-1}}^{\prime j_{f-1}} C_{\tau_{f-1}}^{j_{f}}\left|\Phi_{J}\right\rangle .
$$

Because the projector $\hat{\mathcal{P}}_{p}^{-}$is absent for $p=f$ in Eq.(15), only Step 1 is executed at the rightmost site and now the wavefunction is expressed as

$$
\sum_{\boldsymbol{\tau}, J} L_{\tau_{1}}^{\prime j_{1}} L_{\tau_{1} \tau_{2}}^{\prime j_{2}} \cdots L_{\tau_{f-2} \tau_{f-1}}^{\prime j_{f-1}} C_{\tau_{f-1}}^{\prime j_{f}}\left|\Phi_{J}\right\rangle
$$

This is the end of the former half propagation with a left-to-right sweep, then the latter half propagation will be done in the opposite direction, i.e. a right-to-left sweep, in the steps below.

6) The same as Step 1 the coefficient of the current site $C_{\tau_{p-1} \tau_{p}}^{\prime j_{p}}$ is propagated to $C_{\tau_{p-1} \tau_{p}}^{\prime \prime j_{p}}$ by applying the operator exponential $e^{-i \hat{\mathcal{P}}_{p}^{+} \hat{H} t / 2}$. 
7) The wavefunction after the propagation in the previous step is expressed as

$$
\sum_{\tau, J} L_{\tau_{1}}^{\prime j_{1}} \cdots L_{\tau_{p-2} \tau_{p-1}}^{\prime j_{p-1}}\left(C_{\tau_{p-1} \tau_{p}}^{\prime \prime j_{p}}\right) R_{\tau_{p} \tau_{p+1}}^{\prime \prime j_{p+1}} \cdots R_{\tau_{f-1}}^{\prime \prime j_{f}}\left|\Phi_{J}^{\alpha}\right\rangle .
$$

It is transformed to

$$
\sum_{\tau, \gamma_{p}, J} L_{\tau_{1}}^{\prime j_{1}} \cdots L_{\tau_{p-2} \tau_{p-1}}^{\prime j_{p-1}}\left(\sigma_{\gamma_{p-1}}^{\prime \prime \tau_{p-1}} R_{\gamma_{p-1} \tau_{p}}^{\prime \prime j_{p}}\right) R_{\tau_{p} \tau_{p+1}}^{\prime \prime j_{p+1}} \cdots R_{\tau_{f-1}}^{\prime \prime j_{f}}\left|\Phi_{J}^{\alpha}\right\rangle
$$

by using the orthogonal decomposition in Eq.(8)

8) The same as Step 33 the matrix $\sigma_{\tau_{p-1} \gamma_{p-1}}^{\prime \prime}$ is propagated backward in time to $\sigma_{\tau_{p-1} \gamma_{p-1}}^{\prime}$ by applying the operator exponential $e^{+i \hat{\mathcal{P}}_{p-1}^{-} \hat{H} \delta}$.

9) Similar to Step 团 the wavefunction expressed as

$$
\sum_{\tau, \gamma_{p}, J} L_{\tau_{1}}^{\prime j_{1}} \cdots\left(L_{\tau_{p-2} \gamma_{p-1}}^{\prime j_{p-1}} \sigma_{\tau_{p-1}}^{\prime \gamma_{p-1}}\right) R_{\tau_{p-1} \tau_{p}}^{\prime \prime j_{p}} \cdots R_{\tau_{f-1}}^{\prime \prime j_{f}}\left|\Phi_{J}^{\alpha}\right\rangle
$$

is transformed to

$$
\sum_{\tau, J} L_{\tau_{1}}^{\prime j_{1}} \cdots\left(C_{\tau_{p-2} \tau_{p-1}}^{\prime j_{p-1}}\right) R_{\tau_{p-1} \tau_{p}}^{\prime \prime j_{p}} \cdots R_{\tau_{f-1}}^{\prime \prime j_{f}}\left|\Phi_{J}^{\alpha}\right\rangle
$$

10) Step 69 are repeated until the current site $p$ reaches the leftmost site $p=1$

$$
\sum_{\tau, J} C_{\tau_{1}}^{\prime j_{1}} R_{\tau_{1} \tau_{2}}^{\prime \prime j_{2}} \cdots R_{\tau_{f-2} \tau_{f-1}}^{\prime \prime j_{f-1}} R_{\tau_{f-1}}^{\prime \prime j_{f}}\left|\Phi_{J}\right\rangle
$$

Lastly, the coefficient $C_{\tau_{1}}^{\prime j_{1}}$ is propagated to $C_{\tau_{1}}^{\prime \prime j_{1}}$ in the same way as Step 6.

\section{APPENDIX B: COMPARISON WITH THE MULTILAYER FORMULATION}

A different form of the equation of motion (EOM) for the MPS-MCTDH wavefunction ansatz defined in Eq.(1) with Eq.(2) and (3) can be derived in the framework of the multi-layer formulation, [27, 29, 30] in which an immutable ML-tree structure is defined, e.g. if we choose the site coefficient at $p$, namely $A_{\tau_{p-1} \tau_{p}}^{j_{p}}$, as the top layer coefficient, the definition of the ML-tree is given as

Top layer

$$
|\Psi\rangle=A_{\tau_{p-1} \tau_{p}}^{(1) j_{p}}\left|\Psi_{\tau_{p-1}}^{\mathrm{L}} \varphi_{j_{p}} \Psi_{\tau_{p}}^{\mathrm{R}}\right\rangle,
$$

Left tree

$$
\begin{aligned}
\left|\Psi_{\tau_{p-1}}^{\mathrm{L}}\right\rangle & =A_{\tau_{p-2} \tau_{p-1}}^{(2) j_{p-1}}\left|\Psi_{\tau_{p-2}}^{\mathrm{L}} \varphi_{j_{p-1}}\right\rangle, \\
\left|\Psi_{\tau_{p-2}}^{\mathrm{L}}\right\rangle & =A_{\tau_{p-3} \tau_{p-2}}^{(3) j_{p-2}}\left|\Psi_{\tau_{p-3}}^{\mathrm{L}} \varphi_{j_{p-2}}\right\rangle, \\
& \vdots \\
\left|\Psi_{\tau_{2}}^{\mathrm{L}}\right\rangle & =A_{\tau_{1} \tau_{2}}^{(p-1) j_{2}}\left|\Psi_{\tau_{1}}^{\mathrm{L}} \varphi_{j_{2}}\right\rangle, \\
\left|\Psi_{\tau_{1}}^{\mathrm{L}}\right\rangle & =A_{\tau_{1}}^{(p) j_{1}}\left|\varphi_{j_{1}}\right\rangle,
\end{aligned}
$$

Right tree

$$
\begin{aligned}
\left|\Psi_{\tau_{p}}^{\mathrm{R}}\right\rangle & =A_{\tau_{p} \tau_{p+1}}^{(2) j_{p+1}}\left|\varphi_{j_{p+1}} \Psi_{\tau_{p+1}}^{\mathrm{R}}\right\rangle, \\
\left|\Psi_{\tau_{p+1}}^{\mathrm{R}}\right\rangle & =A_{\tau_{p+1} \tau_{p+2}}^{(3) j_{p+2}}\left|\varphi_{j_{p+2}} \Psi_{\tau_{p+2}}^{\mathrm{R}}\right\rangle, \\
\vdots & \\
\left|\Psi_{\tau_{f-2}}^{\mathrm{R}}\right\rangle & =A_{\tau_{f-2} \tau_{f-1}}^{(f-p) j_{f-1}}\left|\varphi_{j_{f-1}} \Psi_{\tau_{f-1}}^{\mathrm{R}}\right\rangle, \\
\left|\Psi_{\tau_{f-1}}^{\mathrm{R}}\right\rangle & =A_{\tau_{f-1}}^{(f-p+1) j_{f}}\left|\varphi_{j_{f}}\right\rangle,
\end{aligned}
$$

where the parenthesis in the super script denotes the depth in the ML-tree structure. In the standard ML-MCTDH notation, [58] $A_{\tau_{p-3} \tau_{p-2}}^{(3) j_{p-2}}$ is usually noted as $A_{\tau_{p-2} ; \tau_{p-3}, j_{p-2}}^{3 ; \mathrm{L}}$ where $\mathrm{L}$ in the superscript denotes the route from the root to the node under consideration, but there are only two routes (L or R) in the MPS ansatz. In the ML-formulation, the generalized SPFs $\Psi^{\mathrm{L}(\mathrm{R})}$ at every layer should be kept in orthonormal which is equivalent to fix the representation of the wavefunction in the specific $p$-canonical form of the MPS written as

$\sum_{\boldsymbol{\tau}, J} L_{\tau_{1}}^{(p) j_{1}} \cdots L_{\tau_{p-2} \tau_{p-1}}^{(2) j_{p-1}} C_{\tau_{p-1} \tau_{p}}^{(1) j_{p}} R_{\tau_{p} \tau_{p+1}}^{(2) j_{p+1}} \cdots R_{\tau_{f-1}}^{(f-p+1) j_{f}}\left|\Phi_{J}\right\rangle$,

throughout the propagation, which is in contrast to the MPS-formulation, in which the gauges of the site coefficients are consecutively changed as Eq. (8) and the representation for the wavefunction is transformed between the canonical forms of the different sites. In the ML-formulation, because the representation of the wavefunction is fixed as the canonical form of the specific site $p$, the basis states for the generalized single hole functions (SHF) are non-orthogonal except for the top layer and the inversion of the overlap matrices of the SHFs, which can be singular sometimes, appear in the EOMs for the lower layers, whereas in the MPS-formulation the wavefunction is always expressed by the direct products of the orthonormal basis states at every node of the tree and the inverse matrices are completely eliminated in the EOMs.

The time propagation with the EOMs derived in the MPS-formulation is, therefore, robust even for the systems with many layers. Such systems can be found in an interesting application 18 in which infinite bath modes are mapped onto an effective 1-D chain modes to efficiently simulate the open quantum dynamics beyond 
the perturbation theory. 54, 55] In addition, in contrast to the standard ML-formulation, the MPS-formulation with the tangent space projector splitting method allows us to adopt the Lanczos integrator, which possesses favorable properties, 23] for the time propagation of the site coefficients in spite of the highly non-linear parametrization of the MPS ansatz, because the differential equations obtained from the individual split tangent space projectors are linear equations and exactly solvable. The accurate time-integration algorithm should allow a comparatively large step size for the propagation of the site coefficients, namely the MCTDH expansion coefficients.

There is an ongoing argument regarding the pros and cons of the projector-splitting integrator applied to the EOMs of the SPF in the conventional MCTDH. 41] 43, 56, 57 While it can remove the inversion of the density matrix completely from the EOMs and is robust and not collapsed even when the density matrix has zero eigenvalues caused by the presence of unoccupied natural orbitals, the propagation of the unoccupied orbitals determined by the projector splitting integrator will be somewhat arbitrary because it involves orthogonal decompositions of the density matrix. This behavior is a consequence of the nature of the first-order equation, and to be accurate, it is not correct for the second-order in time as discussed in detail by Manthe in Ref [56]. In the standard MCTDH implementation, the problem of this inaccurate motion of the unoccupied orbitals is usually mitigated by the regularization of the density matrix, 23. which introduces an artificial occupation to the unoccupied orbitals with a small number $\varepsilon$ primarily to avoid the non-invertible density matrix problem, since these orbitals occupied by the small number $\varepsilon$ are expected to be rotated quickly into their correct direction due to the so-called self-healing effect of the MCTDH. In fact, the recently developed new regularization scheme, 57, 58] which allows a much smaller value for $\varepsilon$ comparing with the conventional scheme, has exhibited an ability to rotate the unoccupied orbitals more quickly to the correct directions. The MPS-formulation in this paper also adopts the projector-splitting integrator method and the situation seems to be much the same as described above, for instance, if there is an unoccupied state as $w_{\tau_{p}}^{(\mathrm{L})}=0$ in Eq.(10), the transformation to the state $\Psi_{\tau_{p+1}}^{\mathrm{L}}$ becomes non-unique, and thus further investigation is needed on that point.

[1] S. R. White, Phys. Rev. Lett. 69, 2863 (1992).

[2] S. R. White, Phys. Rev. B 48, 10345 (1993).

[3] S. R. White and R. L. Martin, J. Chem. Phys. 110, 4127 (1999).

[4] G. K.-L. Chan and M. Head-Gordon, J. Chem. Phys. 116, 4462 (2002).
[5] S. R. White and A. E. Feiguin, Phys. Rev. Lett. 93, 076401 (2004).

[6] A. J. Daley, C. Kollath, U. Schollwöck, and G. Vidal, J. Stat. Mech. 2004, P04005 (2004).

[7] G. Vidal, Phys. Rev. Lett. 93, 040502 (2004).

[8] J. Haegeman, J. I. Cirac, T. J. Osborne, I. Pižorn, H. Verschelde, and F. Verstraete, Phys. Rev. Lett. 107, 070601 (2011).

[9] C. Lubich, T. Rohwedder, R. Schneider, and B. Vandereycken, SIAM J. Matrix Anal. Appl. 34, 470 (2013).

[10] J. Haegeman, T. J. Osborne, and F. Verstraete, Phys. Rev. B 88, 075133 (2013).

[11] K. Ueda, C. Jin, N. Shibata, Y. Hieida, and T. Nishino, arXiv (2006), cond-mat/0612480v2

[12] J. J. Dorando, J. Hachmann, and G. K.-L. Chan, J. Chem. Phys. 130, 184111 (2009).

[13] J. M. Kinder, C. C. Ralph, and G. K.-L. Chan, arXiv (2011), 1103.2155v1

[14] J. Kinder, C. Ralph, and G. Chan, Advances in Chemical Physics 154, 179 (2014).

[15] S. Wouters, N. Nakatani, D. Van Neck, and G. K.-L. Chan, Phys. Rev. B 88, 075122 (2013).

[16] N. Nakatani, S. Wouters, D. Van Neck, and G. K.-L. Chan, J. Chem. Phys. 140, 024108 (2014).

[17] J. Haegeman, C. Lubich, I. Oseledets, B. Vandereycken, and F. Verstraete, Phys. Rev. B 94, 165116 (2016).

[18] F. A. Y. N. Schröder and A. W. Chin, Phys. Rev. B 93, 075105 (2016).

[19] R. Borrelli and M. F. Gelin, Sci. Rep. , 1 (2017).

[20] B. Kloss, Y. B. Lev, and D. Reichman, Phys. Rev. B , 1 (2018).

[21] H.-D. Meyer, U. Manthe, and L. S. Cederbaum, Chem. Phys. Lett. 165, 73 (1990).

[22] U. Manthe, H.-D. Meyer, and L. S. Cederbaum, J. Chem. Phys. 97, 3199 (1992).

[23] M. H. Beck, A. Jäckle, G. A. Worth, and H.-D. Meyer, Phys. Rep. 324, 1 (2000).

[24] G. A. Worth, H.-D. Meyer, and L. S. Cederbaum, J. Chem. Phys. 105, 4412 (1996).

[25] A. Raab, G. A. Worth, H.-D. Meyer, and L. S. Cederbaum, J. Chem. Phys. 110, 936 (1999).

[26] L. Grasedyck, SIAM J. Matrix Anal. Appl. 31, 2029 (2010).

[27] U. Manthe, J. Chem. Phys. 128, 164116 (2008).

[28] H. Wang, J. Phys. Chem. A 119, 7951 (2015).

[29] H. Wang and M. Thoss, J. Chem. Phys. 119, 1289 (2003).

[30] O. Vendrell and H.-D. Meyer, J. Chem. Phys. 134, 044135 (2011).

[31] H. Wang and M. Thoss, J. Phys. Chem. A 111, 10369 (2007).

[32] L. Cao, S. Krönke, O. Vendrell, and P. Schmelcher, J. Chem. Phys. 139, 134103 (2013).

[33] U. Manthe, J. Phys.: Condens. Matter 29, 253001 (2017).

[34] U. Manthe and T. Weike, J. Chem. Phys. 146, 064117 (2017).

[35] O. Kühn and S. Lochbrunner, arXiv (2011), $1108.4834 \mathrm{v} 2$

[36] M. Schröter, S. D. Ivanov, J. Schulze, S. P. Polyutov, Y. Yan, T. Pullerits, and O. Kuhn, Phys. Rep. 567, 1 (2015).

[37] J. Schulze, M. F. Shibl, M. J. Al-Marri, and O. Kühn, J. Chem. Phys. 144, 185101 (2016).

[38] M. F. Shibl, J. Schulze, M. J. Al-Marri, and O. Kühn, 
J. Phys. B: At. Mol. Opt. Phys. 50, 184001 (2017).

[39] Y. Y. Shi, L. M. Duan, and G. Vidal, Phys. Rev. A 74, 134 (2006).

[40] U. Schollwöck, Ann. Phys. 326, 96 (2011).

[41] C. Lubich, Appl. Math. Res. Express 2015, 311 (2015).

[42] B. Kloss, I. Burghardt, and C. Lubich, J. Chem. Phys. 146, 174107 (2017).

[43] M. Bonfanti and I. Burghardt, ArXiv e-prints (2018), arXiv:1802.01058 [physics.chem-ph].

[44] M. H. Beck and H.-D. Meyer, Z. Phys. D 42, 113 (1997).

[45] S. Carter, S. J. Culik, and J. M. Bowman, J. Chem. Phys. 107, 10458 (1997).

[46] K. Yagi, K. Hirao, T. Taketsugu, M. W. Schmidt, and M. S. Gordon, J. Chem. Phys. 121, 1383 (2004).

[47] Y. Kurashige and T. Yanai, J. Chem. Phys. 135, 094104 (2011)

[48] H. Tamura, E. R. Bittner, and I. Burghardt, J. Chem. Phys. 126, 021103 (2007).
[49] H. Tamura, R. Martinazzo, M. Ruckenbauer, and I. Burghardt, J. Chem. Phys. 137, 22A540 (2012).

[50] H. Tamura, M. Huix-Rotllant, I. Burghardt, Y. Olivier, and D. Beljonne, Phys. Rev. Lett. 115, 107401 (2015).

[51] R. Binder, M. Polkehn, T. Ma, and I. Burghardt, Chem. Phys. 482, 16 (2017).

[52] J. Ren, Z. Shuai, and G. K.-L. Chan, arXiv (2018), 1806.07443.

[53] Y. Kurashige and T. Yanai, J. Chem. Phys. 130, 234114 (2009).

[54] J. Prior, A. W. Chin, S. F. Huelga, and M. B. Plenio, Phys. Rev. Lett. 105, 050404 (2010).

[55] R. Rosenbach, J. Cerrillo, S. F. Huelga, J. Cao, and M. B. Plenio, New J. Phys. 18, 023035 (2016).

[56] U. Manthe, J. Chem. Phys. 142, 244109 (2015).

[57] H.-D. Meyer and H. Wang, J. Chem. Phys. 148, 124105 (2018).

[58] H. Wang and H.-D. Meyer, J. Chem. Phys. 149, 044119 (2018). 